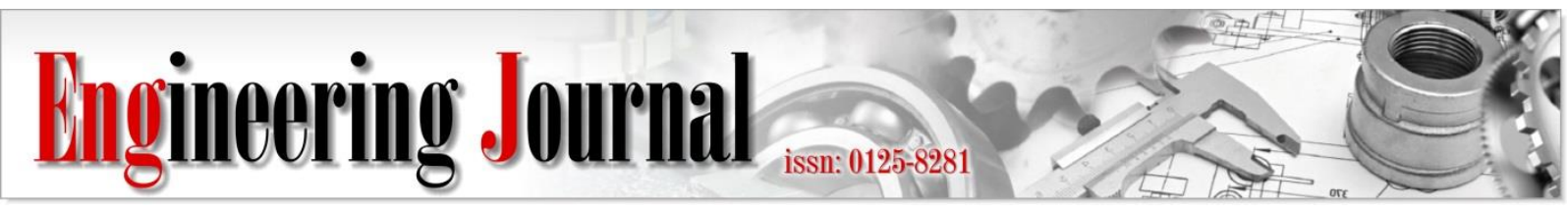

Article

\title{
Investigation of the Electronic Thermal Transport in Thin Silicon Films with Varying Electric Field and Doping Concentration
}

\author{
Jae Sik Jin \\ Department of Mechanical Design, Chosun College of Science \& Technology, Gwangju 61453, Republic of \\ Korea \\ E-mail: jinjs@cst.ac.kr (Corresponding author)
}

\begin{abstract}
Scatterings of electrons by ionized impurities in semiconductors have been comprehensively explored by various methods. However, the electron-impurity scattering was not investigated under an electric field at thin silicon ( $\mathrm{Si}$ ) films. The effects of electric field on electronic thermal conductivity in thin Si films at different doping concentration at room temperature are investigated by using an analytical model based on the Boltzmann transport equation with the relaxation time approximation. As expected, the electronic thermal conductivity increases as the external electric field increases. More importantly, we show that the scattering between carrier and impurity is a dominant scattering mechanism in Si films at high doping level such as above $10^{18} \mathrm{~cm}^{-3}$ under the influence of an electric field. The effect of the electron-phonon interaction on the electronic thermal conductivity of silicon can be neglected because electron mean free path is significantly reduced due to the impurity scattering and the effect of electric field. Since there is no experimental attempt to measure the electron and the phonon thermal conductivities with the electric field applied, the result is given of some simplified concepts that will contribute to a better understanding of electron transport fundamentals.
\end{abstract}

Keywords: Electronic thermal conductivity, electric field, doping concentration, Boltzmann transport equation, thin silicon film.

ENGINEERING JOURNAL Volume 24 Issue 3

Received 2 October 2019

Accepted 24 April 2020

Published 31 May 2020

Online at https://engj.org/

DOI:10.4186/ej.2020.24.3.149 


\section{Introduction}

After the introduction of nanotechnology, MicroElectro Mechanical Systems (MEMS) fabrication makes a thin silicon (Si) film a useful material in many devices such as sensor, actuator, and electric devices [1]. Because phonons play a major role in the thermal transport of $\mathrm{Si}$, phonon manipulation is the most interesting topic in the design of Si-based electronics devices for engineering applications [1-4]. Although a bulk $\mathrm{Si}$ has a good thermal conductivity $(\sim 148 \mathrm{~W} / \mathrm{mK}$ at $300 \mathrm{~K})$, the lateral thermal conductivity of a sub- $100 \mathrm{~nm}$ thick Si layer, which is used extensively for MEMS process, can be reduced to the value of several 10 $\mathrm{W} / \mathrm{mK}$ at $300 \mathrm{~K}[2,3]$. This significantly reduced thermal conductivity is mainly caused by reduced mean-free paths of phonons due to the phonon-boundary scatterings.

It has been well known that phonon transport in the materials might be influenced by the processes of the electron-phonon interactions [2, 3]. Therefore, understanding electron-phonon interaction is very important in the study of thermoelectrics [4], superconductivity $[5,6]$, heat generation problems in electronic devices $[7,8]$, and phonon transfer regulation [9]. However, the fundamental understanding of both electrical and thermal transport with an electric field is rather limited: for example, Bolin et al. [4] showed a significant reduction of the lattice thermal conductivity at room temperature as the electron concentration goes above $10^{19} \mathrm{~cm}^{-3}$ and the reduction reaches up to $45 \%$ in p-type $\mathrm{Si}$ at around $10^{21} \mathrm{~cm}^{-3}$. However, they did not consider the electric field effect. And really fewer studies are conducted to investigate the electric field effect on both electron and phonon transport, especially in a thin Si film.

At sub-micron length scales, the conventional heat diffusion (Fourier's law) cannot be employed because both the ballistic phonon transport and the phononboundary scattering become dominant, and the relaxation time of energy carriers is finite $[1,7]$. The thermal behavior of energy carriers such as phonons and electrons can be investigated by using the Boltzmann transport equation (BTE) in the regime where the particle description of energy carriers is valid $[1,7]$. Recently, as a computational tool for studying heat conduction in nanostructures, the electron-phonon interaction model was developed and widely applied to calculate the thermal properties in $\mathrm{Si}$ based devices during the last decade $[7,8,10,11]$. Among the works, the thermal conductions of each energy carrier (phonon and electron) in $\mathrm{Si}$ were investigated by using a modified version of the electron-phonon interaction model with varying the doping concentration and the Si-layer thickness [11].

Doping process is extensively used in semiconductor industries to improve the electrical characteristics of Si by adding impurities into an intrinsic Si intentionally. For example, the carrier concentrations must be manipulated to achieve the n-type and $\mathrm{p}$-type semiconductors employed in the fabrication of devices: The source and drain regions of transistor are highly doped (above $\sim 10^{20}$ $\mathrm{cm}^{-3}$ ) with dopants to augment electrical conduction by increasing carrier concentration [11]. As mentioned before, the thermal conductivities of $\mathrm{Si}$ based nanostructures can be reduced by one or two orders of magnitude from the bulk value due to the phononboundary scattering. In addition to this boundaryphonon scattering, the implanted impurity atoms also deteriorate the thermal conductivities of thin $\mathrm{Si}$ films due to increasing phonon-impurity scattering events [11]. Electron, which is a kind of energy carrier, is also in the same situation. Therefore, for the development of efficient electronic devices, a study on the movement of electrons in silicon-based electronic devices is important.

To the best of our knowledge, there has no study of the electric field dependent electron transport in Si films at different doping concentrations. In the present study, the phosphorus that is the most representative n-type dopant is used as a doped impurity in thin Si films, and the electric field effects is investigated to estimate carrier conduction in Si layer by using a revised electron-phonon interaction model. Actually, the electric field has a different local distribution even at the same doping level depending on the doping distribution configuration. But this effect is neglected in this study for the sake of simplification. However, the data provided here can be used for the fundamental understandings of a thermal energy transport in a highly doped region of electronic devices.

\section{Revised Forms of Electron-Phonon Interaction Model}

\subsection{Phonon Boltzmann Transport Equation}

In order to calculate thermal energy transfer between phonons and electrons, we employed an analytical model, the electron-phonon interaction model that is based on the phonon Boltzmann transport equation. The details of the important physical concept of the electron-phonon interaction model have been described elsewhere $[7,8,10$, 11]. Here we start from the version used in Ref. 11 to calculate the phonon's contributions to thermal conductivity in $\mathrm{Si}$ films with varying the doping concentration. Under the relaxation time approximation, the phonon BTE's for each acoustic and optical mode are shown as [11]:

$$
\begin{aligned}
\frac{\partial e_{i}^{\prime \prime}}{\partial t}+\nabla \cdot\left(v_{i} \hat{s} e_{i}^{\prime \prime}\right) & =\left(e_{i}^{o}-e_{i}^{\prime \prime}\right)\left(\gamma_{i i}+\gamma_{i m p, i}\right) \\
& +\sum_{\substack{j=1 \\
j \neq i}}^{N_{\text {banns }}}\left\{\left(\frac{1}{4 \pi} \int_{T_{\text {ref }}}^{T_{i j}} C_{i} d T-e_{i}^{\prime \prime}\right) \gamma_{i j}\right\} \\
& +\left(\int_{T_{\text {ref }}}^{T_{e i}} C_{e} d T-e_{e}^{\prime \prime}\right) \gamma_{\text {int or }(f \text { or } g)}
\end{aligned}
$$




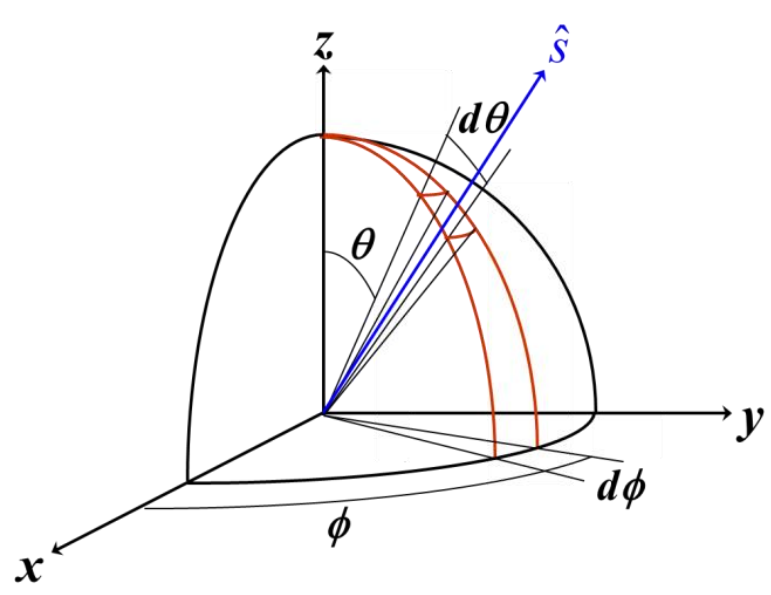

Fig. 1. Coordinate system and definition of phonon direction $\hat{s}$. In the figure, $\theta$ and $\phi$ denote the polar angle and the azimuthal angle, respectively.

$$
\begin{aligned}
\frac{\partial e_{o}}{\partial t} & =\sum_{j=1}^{N_{\text {bands }}-1}\left(\int_{T_{r e f}}^{T_{o j}} C_{o} d T-e_{o}\right) \gamma_{o j}+\left(\int_{T_{r e f}}^{T_{e o}} C_{e} d T-e_{o}\right) \gamma_{g} \\
& +\sum_{f=1}^{2}\left(\int_{T_{r e f}}^{T_{e o}} C_{e} d T-e_{o}\right) \gamma_{f}
\end{aligned}
$$

The definition of all the physical parameters used in the above equations can be found in Ref. 11: In short, $e_{e}$ is the electron energy concentration, $C_{i}, C_{o}$, and $C_{e}$ are the specific heats of $i$-th acoustic and optical phonon bands, and the electrons, respectively. $T_{r e f}$ is the reference temperature (here, $T_{r e f}=300 \mathrm{~K}$ ), and $v_{i}$ is the $i$-th phonon group velocity, which is computed from the phonon dispersion relation $\left(v_{i}=\partial \omega_{i} / \partial k_{i}\right.$ where $\omega_{i}$ and $k_{i}$ are the $i$-th phonon frequency and wavenumber, respectively). $\hat{s}$ is the unit direction vector (see Fig. 1 for details). In this work, the effect of doping concentration on the phonon dispersion relation is not considered for the sake of simplicity. $e_{i}^{\prime \prime}$ and $e_{0}$ are the volumetric energy densities for a given acoustic band for each direction $(\hat{s})$ and for optical band, respectively. $e_{i}^{0}$ is the equilibrium energy concentration of phonons. $T_{i j}$ and $T_{o j}$ are the interaction temperatures among acoustic ( $i$ and $j$ ) phonons and optical (o) phonon, $\gamma_{i j}, \gamma_{i i}$ and $\gamma_{o j}$ are the scattering rates for each scattering process. $T_{e j}$ is the interaction temperature corresponding to the electron and acoustic phonon interaction $\left(T_{e a}\right)$ or optical phonon interaction $\left(T_{e o}\right) . N_{\text {bands }}$ is the total number of frequency bands, i.e. $N_{\text {bands }}=N_{L A}+N_{T A}+1$, where $N_{L A}, N_{T A}$, and unity are the number of frequency bands in longitudinal and transverse acoustic phonons and optical phonons, respectively (in this work, $N_{L A}=N_{T A}=6$ as used in Refs. 7,8 , and 11). The impurity scattering rates $\gamma_{i m p, i}$ is incorporated into the first term on the right hand side of Eq. (1) to account for the effects of inelastic scattering between doped impurity and phonon. Also $\gamma_{\text {int }}, \gamma_{\mathrm{f}}$, and $\gamma_{\mathrm{g}}$ represent the scattering rates corresponding to intravalley interaction, $f$-process, and $g$-process through intervalley

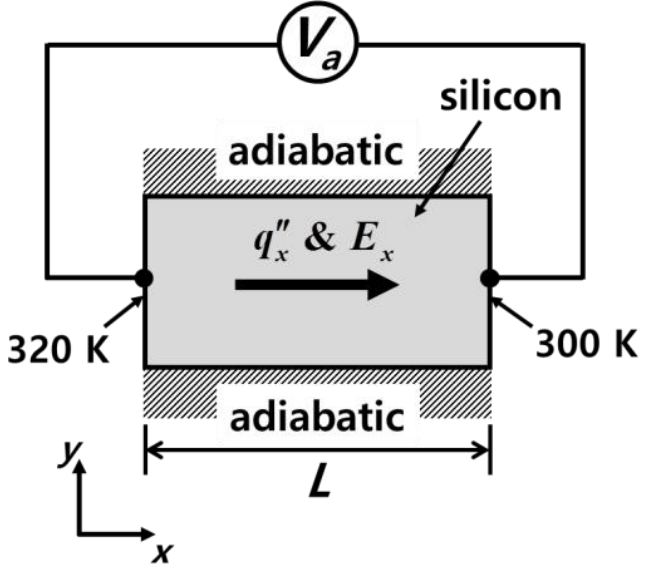

Fig. 2. Schematic of calculation domain with the heat flux $\left(q_{x}^{\prime \prime}\right)$ caused by the thermal gradient $(d T / d x)$ and the electric field $\left(E_{x}\right)$ caused by the applied voltage $\left(V_{a}\right)$ in the $x$-direction. In this study, Si film's thicknesses $(L)$ considered are 20, 50, $100 \mathrm{~nm}$.

interaction, respectively. Thus, the first term is account for the effects of phonon dispersion and phononimpurity scattering. The second term means the polarization effect of acoustic phonon mode. The third term on the right side of Eq. (1) corresponds to the energy exchange between electron and acoustic phonon mode through intravalley and intervalley processes. For the optical mode BTE, the three terms in the right hand side of Eq. (2) denote phonon polarization effect and $f$ and $g$-processes through intervalley interaction between electron and optical phonon mode, respectively (refer to Refs. 7, 8 and 11 for more detail). The specific heats of each phonon mode $\left(C_{i}\right.$ and $\left.C_{0}\right)$ are used as provided in [12], and the specific heat of electrons $\left(C_{e}\right)$ is calculated by using the expressions shown in Refs. 7 and 8 :

$$
C_{e}=\frac{\pi^{2}}{2}\left(\frac{k_{B} T_{e}}{E_{F}}\right) \eta_{e} k_{B},
$$

where $k_{B}$ is the Boltzmann constant, $E_{F}$ is the Fermi energy, $\eta_{e}$ is the doping concentration.

\subsection{Electron Boltzmann Transport Equation}

Generally, BTE based methods cannot be applied for analyzing electron transport under the high electric field in semiconductors due to the quantum effects such as the intracollisional field effect and the interference effect associated with strong electric fields [13]. According to the study conducted by Sano and Yoshii [14], using the fully quantum mechanical method, the conventional semi-classical description of carrier transport based on the Boltzmann transport equation breaks down when the electric field is above $\sim \mathrm{MV} / \mathrm{m}$. In this study, the maximum strength of the electric field considered here is only up to $4 \mathrm{MV} / \mathrm{m}$ so that $\mathrm{BTE}$ based model could be applicable to our problems. Moreover, as discussed by the author [8], with the assumption that the electron relaxation times are 
independent of the strength and type of the perturbation, the relaxation time approximation is valid for the electron BTE.

Now in order to include the effects of an electric field and impurity scattering, the original expression for the electron BTE represented in Ref. 11 has to be modified because the thermal gradient was only considered in Ref. 11. The Si film layer that is subjected to both the electric field and the temperature gradient in the $x$-direction is shown in Fig. 2. In order to include the electric field and impurity scattering effects, we should add the external force term into the original formula, and it can be expressed as:

$$
\begin{aligned}
\frac{\partial e_{e}}{\partial t}+\vec{v}_{e} \cdot \nabla e_{e}+\vec{F} \cdot \frac{\partial e_{e}}{\partial \vec{P}} & =e_{e} \cdot \gamma_{i m p, e}+\sum_{a=1}^{5}\left(\int_{T_{r e f}}^{T_{e a}} C_{e} d T-e_{e}\right) \gamma_{i n t} \\
& +\sum_{a=6}^{9}\left(\int_{T_{r e f}}^{T_{e a}} C_{e} d T-e_{e}\right) \gamma_{i n t ~(f \text { or } g)} \\
& +\left(\int_{T_{r e f}}^{T_{e o}} C_{e} d T-e_{e}\right) \gamma_{g} \\
& +\sum_{f=1}^{2}\left(\int_{T_{r e f}}^{T_{e o}} C_{e} d T-e_{e}\right) \gamma_{f}
\end{aligned}
$$

where $\vec{v}_{e}$ is the electron velocity caused by both the thermal gradient and the electric field. $\vec{F}$ is the external force defined as $\vec{F}=e \cdot \vec{E}$ with the electronic charge $e=$ $1.6 \times 10^{-19} \mathrm{C}$ and the electric field $\vec{E}$. $\gamma_{i m p, e}$ is the scattering of electrons by ionized impurities in doped Si. The energy exchange between electron and acoustic or optical phonon mode is represented in the right hand side of Eq. (4). Since the electron-electron scattering is fast enough to restore equilibrium between them [15], the effect of electron-electron interaction can be assumed as negligibly small compared to those of the other energy exchange processes.

\section{Scattering Rates between Energy Carriers}

There may be many kinds of carrier scattering processes in $\mathrm{Si}$ and, therefore, their scatteringmechanisms are very complicated. Due to this inherent complexity of the phenomena involved in energy carrier scatterings, several approximations have been made in this study to simplify the problem. Otherwise, a huge amount of computation time is needed to calculate their scattering rates. For simplicity, the column $\mathrm{V}$ atom "donates" a conduction band electron to the system. Charge balance is nevertheless maintained because the fixed donor site itself becomes charged (ionized) in providing carrier to the system [16].

Note that a surplus electron of phosphorus becomes energy carrier, and charged donor can be treated as an impurity. The scattering rate of these free electrons and phonons can be described by the processes of intravalley and intervalley scatterings [17] that is varied with the electron energy level. In this study, the electron energy is assumed as an average electron energy that will be explained later. This approximation is employed because electronic band structures are complicated with multiple valleys or bands at the same or similar energies. The scattering rates of phonon-phonon interaction we used here are the same values presented in literature of Narumanchi et al. [18], that is, frequency-dependent values are used. The scattering rates between electrons and phonons are used according to the values as provided in Ref. 8. As mentioned before, the charged donors can be treated as impurities, and these scatterings with phonon are modeled simply as in Ref. 11, which is proposed by Mazumder et al. [19]:

$$
\gamma_{i m p, i}=\alpha \sigma_{i} \eta_{\mathrm{e}} v_{i}
$$

where $\alpha$ is a constant set as 50 [11], which can be empirically determined by the best fit to the experimental data, and $\sigma_{i}$ is the scattering cross-section of $i$-th phonon band expressed as [11]:

$$
\sigma_{i}=\pi r^{2}\left[\frac{\left(r k_{i}\right)^{4}}{\left(r k_{i}\right)^{4}+1}\right]
$$

where $r$ is the atomic radius of the impurity. This approach is also applied in estimation of $\gamma_{i m p, e}$ with the electron wave number $K_{e}$ at the Fermi energy $\left(E_{F}\right)$, yielding $K_{e}=\sqrt{2 m^{*} E_{F} / \hbar^{2}}$. In view of the interaction temperature, the phonon-phonon interaction temperatures and the electron-phonon interaction temperatures can be calculated by the same method of Ref. 11.

\section{Simulation Method and Validation}

\subsection{Simulation Method}

Because the detailed methodology and calculation procedures of a set of Eqs. (1) (2) and Eq. (4) designated for Si were presented elsewhere [7, 8, 11], the relevant detail is not repeated here. The finite volume method $[20,21]$ is employed to solve the twodimensional BTE. The spatial domain is discretized into rectangular control volumes, with the angular domain at any spatial point discretized into nonoverlapping control angles; the centroid of each control angle is denoted by the direction vector $\hat{s}$. Each octant is divided into $N_{\theta} \mathrm{x}$ $N_{\phi}$ control angles, where $N$ is the number of grid, subscripts $\theta$ and $\phi$ are the polar angle and azimuthal angle, respectively (see Fig. 1 for details) [22]. Following the work of Ref. 11, energy conservation is enforced over each control volume and control angle. The details of the discretization procedure are described in Refs. 20 and 21 .

In this study, the phonon dispersion relations of bulk $\mathrm{Si}$ at room temperature in the [001] direction is used. As 
shown in Fig. 2, the each side of Si is maintained at 320 $\mathrm{K}$ and $300 \mathrm{~K}$, yielding the heat flux $\left(q_{x}^{\prime \prime}\right)$ and the other sides are assumed to be adiabatic conditions, and then an electric field is applied. Using the finite volume method $[20,21]$, the volumetric energy concentration of each energy carrier (phonon modes and electrons) is obtained by solving Eqs. (1) (2) and Eq. (4), then the heat flux of each energy carrier is calculated from $q_{p h}^{\prime \prime}=\sum_{i=1}^{N_{\text {band }}-1} \int_{4 \pi} v_{i} e_{i}^{\prime \prime} d \Omega$ and $q_{e}^{\prime \prime}=v_{e} e_{e}$ for phonon modes and electrons, respectively. Here $\Omega$ is the solid angle. Now in order to calculate the electron velocity, we use the assumption of nondegenerate semiconductor. With this assumption, the average kinetic energy $\left(E_{k}\right)$ of electrons given by $E_{k}=m^{*}\left|\vec{v}_{e}\right|^{2} / 2$ in any conduction band can be calculated from $E_{k}=3 k_{B} T_{e} / 2$ if we consider nonequilibrium conditions $[15,16]$. Here, $m^{*}$ is the effective mass that is approximated to be $m^{*} \approx m_{d}$ in case of electronic thermal excitation, where $m_{d}$ is the electron concentration of states effective mass given by $m_{d}=\left(m_{t}^{2} m_{l}\right)^{1 / 3}$, where $m_{t}\left(m_{t}=0.196 m_{0}\right)$ and $m_{l}\left(m_{l}=\right.$ $\left.0.916 m_{0}\right)$ denote the transverse and longitudinal electron mass, respectively, and $m_{0}$ is the mass of the free electron [17]. Thus, the electron velocity is given as $3 k_{B} T_{e} / 2=m^{*}\left|\vec{v}_{e}\right|^{2} / 2$, yielding the relation of $\left|\vec{v}_{e}\right|=\sqrt{3 k_{B} T_{e} / m^{*}}$. In calculation of electron velocity, $T_{e}$ is calculated from the below:

$$
e_{e}=\int_{T_{\mathrm{ref}}}^{T_{e}} C_{e} d T
$$

Here the electron specific heat $C_{e}$ can be assumed to be constant within a given temperature range from $T_{r f}$ to $T_{e}$, and then as a result, $T_{e}$ can be calculated from $T_{e}=T_{r f}+$ $e_{e} / C_{e}$. This approach is also employed in calculating the phonon temperatures.

In general, when the external force is just an electric field, it may be assumed that the energy involved in the drift velocity is negligible in comparison with the thermal energy, which implies that the drift velocity is small in comparison with the thermal velocity [23]. In our calculations, the momentum ( $\vec{P}$ ) derivative term $\left(\partial e_{e} / \partial \vec{P}\right)$ in Eq. (4) is modeled as $\vec{v}_{e} / V$, where $V$ is the volume [24]. The angular resolution in the octant is $6 \times 6$ and the spatial grids are chosen as $130 \times 60$ for the $\mathrm{Si}$ layer thickness of $20 \mathrm{~nm}, 130 \times 70$ for $50 \mathrm{~nm}$, and $130 \times$ 80 for $100 \mathrm{~nm}$ with a uniform mesh size. These grids provide converged results within $0.1 \%$ regardless of the mesh size.

Using Fourier's law, the thermal conductivities of the phonon $\left(k_{p b}\right)$ and the electron $\left(k_{e}\right)$ can be calculated from

$$
q_{p h}^{\prime \prime}=-k_{p h} \frac{d T_{p h}}{d x}
$$

and

$$
q_{e}^{\prime \prime}=-k_{e} \frac{d T_{e}}{d x}
$$

respectively. After solving Eqs. (8) and (9), the total thermal conductivity $\left(k_{s i}\right)$ of a Si film can be calculated

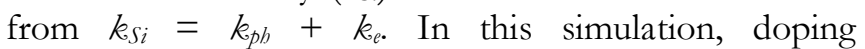
concentration of intrinsic $\mathrm{Si}$ is used as the value of $10^{10}$ $\mathrm{cm}^{-3}$ at room temperature. In our calculations, the average electron energy, $\left\langle E_{e}\right\rangle$, is calculated from $\left\langle E_{e}\right\rangle=0.4 e V_{a}$ proposed by Pop et al. [25]. They simulated the ballistic diode by applied voltage $\left(V_{a}\right)$ of range from 0.2 to $1.0 \mathrm{~V}$ cross the device length of $220 \mathrm{~nm}$, yielding the corresponding values of electric field from $1 \times 10^{6}$ to $4 \times 10^{6} \mathrm{~V} / \mathrm{m}$.

\subsection{Simulation Validation}

The numerical code developed for the present work is verified by comparing with the results of Ref. 11, which neglects the influence of the electric fields. Following the work [11], we calculate three cases with doping of phosphorus atoms: 1) Thermal conductivity of intrinsic Si layer as a function of layer thickness; 2) Thermal conductivity as a function of the doping concentration and the Si layer thickness; 3) Thermal conductivities of each phonon and electron as a function of the doping concentration and Si layer thickness. All calculations are performed at $300 \mathrm{~K}$ in the same degree of specularity used in Ref. 11, and they are in good agreement with previous studies [11] (not shown here). With this validated simulation framework, the influence of electric fields is included and the simulations are performed to investigate the electric field and the doing concentration effects on the electron transport in thin $\mathrm{Si}$ films. Since no experimental study has been conducted to investigate the contributions of the electrons and phonons to the thermal conductivity in the various thicknesses of $\mathrm{Si}$ films with the external electric field applied, we are not able to directly verify our calculations via comparison with any experimental data.

\section{Results and Discussion}

Since metals are usually with the doping concentration well above $10^{22} \mathrm{~cm}^{-3}$, Si still has the character of semiconductors at the doping level applied here even though it is in a heavily doped range of $1 \mathrm{x}$ $10^{18}$ to $5 \times 10^{20} \mathrm{~cm}^{-3}$. Therefore, the thermal conduction is dominated by phonons, and electrons contribute less to the total thermal conductivity in all cases [26]. Now we can calculate the change of $k_{e}$ when the electric field is considered and the doping concentration is varied with changing Si film thickness. In Fig. 3, we show the rate of increase in $k_{e}$ as a function of the electric field and the doping concentration for the cases of Si film's 


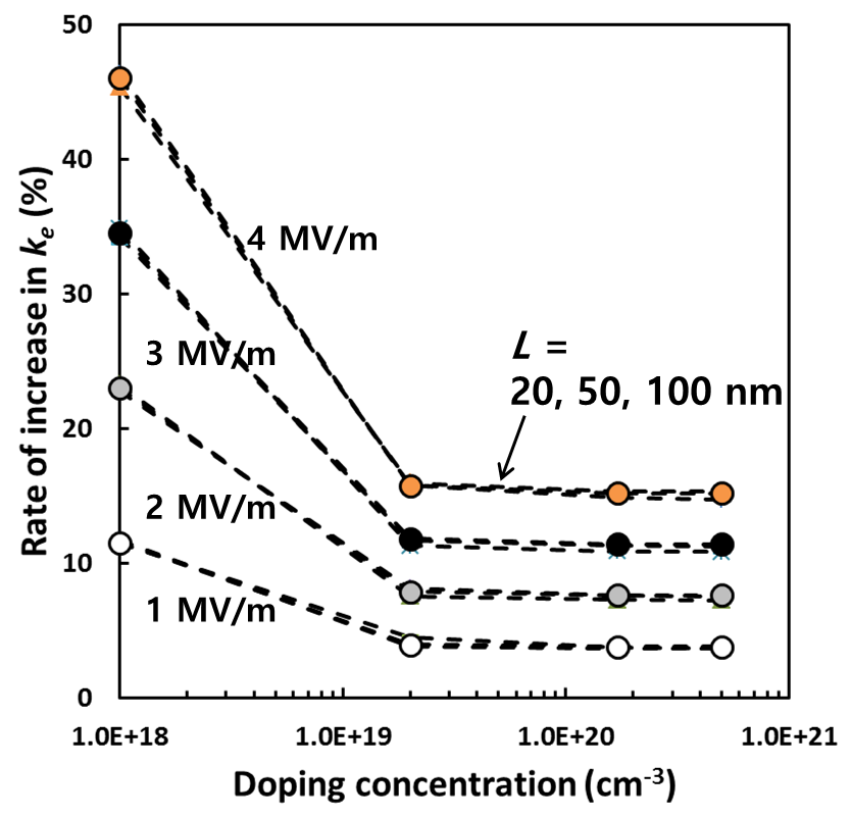

Fig. 3. Rate of increase in the electronic thermal conductivity $\left(k_{e}\right)$ with varying Si film's thicknesses of 20 , 50 , and $100 \mathrm{~nm}$ as a function of electric field and doping concentration. The increase rates of $k_{e}$ for various values of Si film's thicknesses are found to merge nearly into a single curve that it is almost not distinguishable from others.

thicknesses of 20,50 , and $100 \mathrm{~nm}$, showing the increase in $k_{e}$ comparing to the case without the electric fields. From the figure, we can observe several general features. First of all, the electron thermal conductivity increases as the electric field increases, but the degree of increase in $k_{e}$ by the effect of electric field decreases gradually due to the increase of impurity atoms when the doping concentration reaches and over $2 \times 10^{19} \mathrm{~cm}^{-3}$. Since the doping concentration is directly proportional to the amount of impurity atoms, the increased doping concentration definitely leads to decrease in the degree of increase in electronic thermal conductivity due to increasing the impurity scatterings. Unexpectedly, comparing all the cases, the trend of the increase rate of electron thermal conductivity is almost similar and the increase rate of $k_{e}$ for various values of Si film's thickness are found to merge nearly into a single curve that it is almost not distinguishable from others. As a result, we can conclude that the variations of $k_{e}$ caused by both effects of electric field and doping concentration are independent of the thickness change of the Si thin film.

To further analyze the simulation results shown in Fig. 3, the scattering mechanisms of electrons (carriers) in $\mathrm{Si}$ are described schematically in Fig. 4. Electron scattering can be divided into 4 categories: Energy carrier like electron meets the other carrier and bulk mean free path $\left(\Lambda_{\mathrm{B}}\right)$ is determined by such a bulk scattering (see Fig. 4(a)). The other hand, when carriers scatter with a boundary (see Fig. 4(b)), a phonon (see Fig. 4(c)), or an impurity (see Fig. 4(d)) before the bulk scattering occurs, their MFPs will be shorter than $\Lambda_{\mathrm{B}}$. Accordingly, these (a)

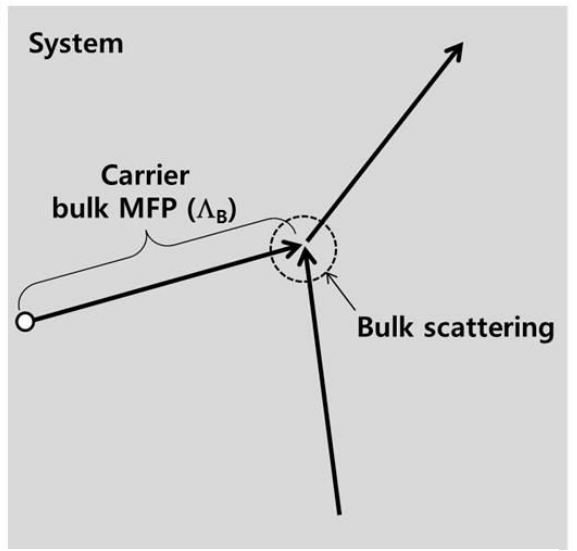

(b)

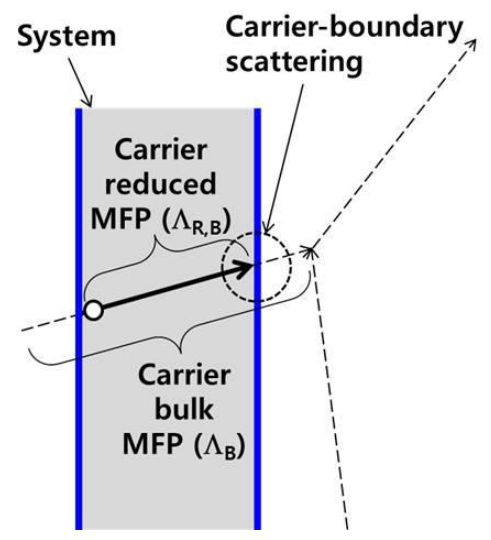

(c)

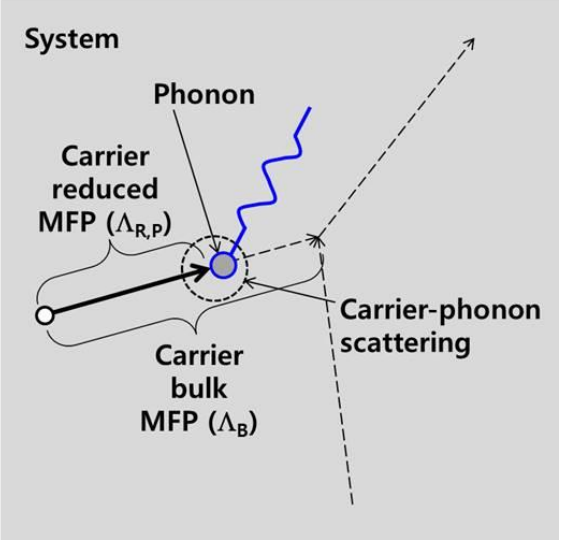

(d)

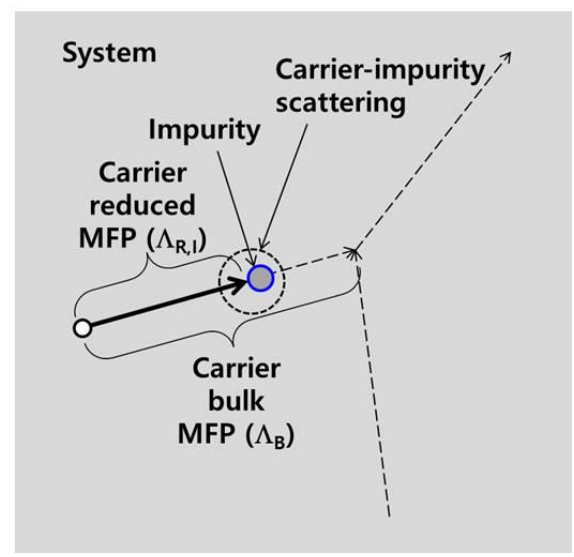

Fig. 4. Scattering mechanisms of electrons (carriers) in Si: (a) Bulk scattering; (b) Carrier-boundary scattering; (c) Carrier-phonon scattering; (d) Carrier-impurity scattering. 


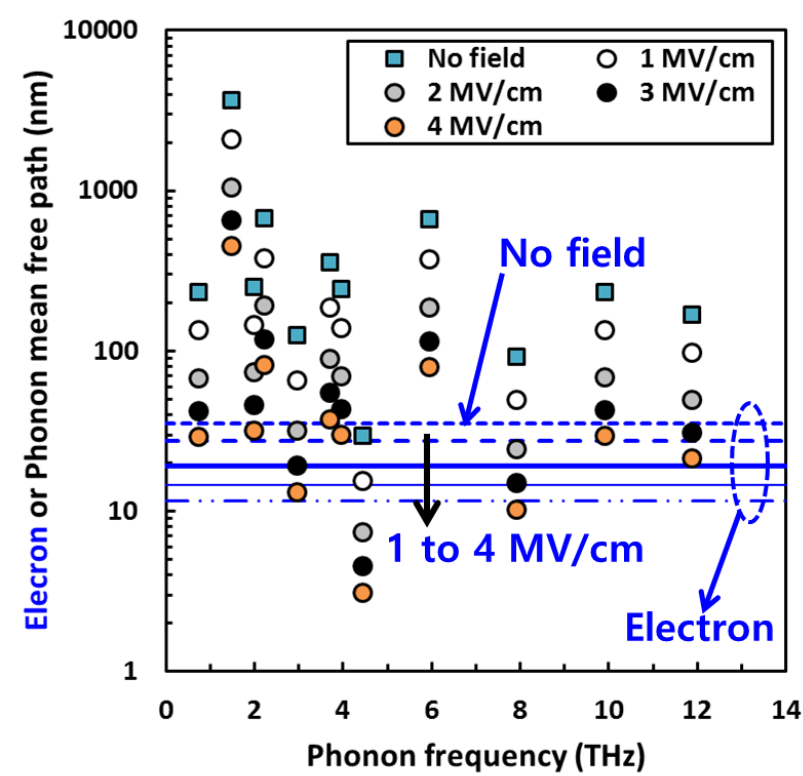

Fig. 5. The MFPs of electrons and phonons in bulk Si with varying electric field applied at the intrinsic doping concentration of $1 \times 10^{10} \mathrm{~cm}^{-3}$ at $300 \mathrm{~K}$.

reduced MFPs (such as $\Lambda_{\mathrm{R}, \mathrm{B}}, \Lambda_{\mathrm{R}, \mathrm{P}}$, and $\Lambda_{\mathrm{R}, \mathrm{I}}$ represented in Fig. 4) result in a reduction in energy transfer by the electrons. In this context, the comparison of each reduced MFP caused by each scattering mechanism becomes an important to answer what scattering mechanism would limit the degree of increase in $k_{e}$. The $\operatorname{MFP}(\Lambda)$ can be calculated from $\Lambda=v / \gamma_{\text {eff }}$ [1]. Here $v$ is either the group velocity for phonons or electron velocity for electrons and $\gamma_{\text {eff }}$ is the effective scattering rate that can be estimated by using Matthiessen's rule [8]. The comparison of MFPs of electrons and phonons in bulk Si with varying electric field at the intrinsic doping concentration of $1 \times 10^{10} \mathrm{~cm}^{-3}$ at $300 \mathrm{~K}$ [16] is shown in Fig. 5. Without electric field case is also represented for comparison. Generally, phonon MFPs without electric field are mainly limited by the anharmonic phononphonon interaction [1]. Here an efficient method for the calculation of electron MFP is employed based on the average electron energy. As the electric field increases, electron scattering rate would increase, and consequently, the electron MFP will be reduced. The change of phonon MFP also shows same trend with varying the electric field applied. As shown in Fig. 5, when an electric field is applied, it can be seen that the minimum length of the electron MFP is $11.9 \mathrm{~nm}$ at $4 \mathrm{MV} / \mathrm{m}$. Figure 6 represents the average distance $(\delta)$ between impurities with varying doping concentrations. Here $\delta$ is calculated from $\left\{4 \pi(\delta / 2)^{3} / 3\right\} \cdot \eta_{e}=1$ in a unit volume of $1 \mathrm{~cm}^{3}$. As shown in the figure, $\delta$ decreases significantly with an increase in $\eta_{e}$, and the maximum MFP is $12.4 \mathrm{~nm}$ that is much shorter than the Si film thickness of $20 \mathrm{~nm}$ within the doping concentration range employed in this study. Since this short $\delta$ limits energy transfer by electrons, the

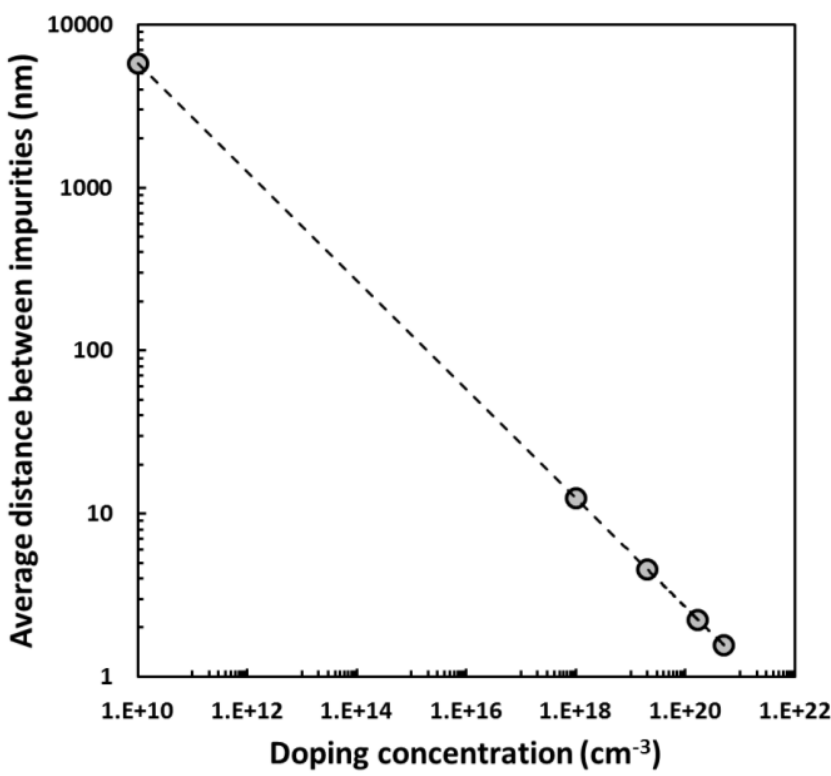

Fig. 6. Average distance between impurities as a function of doping concentration. The dotted line is only eye guide.

change in film thickness does not affect the degree of $k_{e}$ increase as shown in Fig. 3. In conclusion, we show that the carrier-impurity scattering is a dominant scattering mechanism in Si films, which has improved electrical properties by doping, under the influence of an electric field.

\section{Conclusion}

The effects of electric field and doping concentration on electronic thermal conductivity in thin Si films at room temperature are investigated by using an analytical model based on the Boltzmann transport equation (BTE) with the relaxation time approximation. As expected, the electronic thermal conductivity increases as the external electric field increases. It is generally mentioned that since lattice vibrations (i.e., phonons) are enhanced with increasing temperature, electron-phonon scattering usually dominates the scattering process at high temperatures; while at low temperatures, lattice vibrations are weak and defect scattering becomes important. But we show that the scattering between carrier and impurity is a dominant scattering mechanism in Si films at high doping level such as above $10^{18} \mathrm{~cm}^{-3}$ under the influence of an electric field. The effect of the electron-phonon interaction on the electronic thermal conductivity of Si can be neglected because electron mean free path is significantly reduced due to the impurity scattering and the effect of electric field. Since there is no experimental attempt to measure the electron and the phonon thermal conductivities with the electric field applied, the result is given of some simplified concepts that will contribute to a better understanding of electron transport fundamentals. As a future study, it would be a valuable work to investigate whether the variation in electron thermal conductivity under the 
thermal gradient and the electric field can be explained through the amount of scattering term in BTE.

\section{References}

[1] G. Chen, Nanoscale Energy Transport and Conversion, Oxford University Press, Inc., 2005.

[2] J.-T. Lü, H. Zhou, J.-W. Jiang, and J.-S. Wang, "Effects of electron-phonon interaction on thermal and electrical transport through molecular nanoconductors," AIP $A d v$., vol. 5, no. 5, pp. 053204, 2015.

[3] F. Giustino, "Electron-phonon interactions from first principles," Rev. Mod. Phys., vol. 89, no. 1, pp. 15003, 2017.

[4] B. Liao, B. Qiu, J. Zhou, S. Huberman, K. Esfarjani, and G. Chen, "Significant reduction of lattice thermal conductivity by the electron-phonon interaction in silicon with high carrier concentrations: A first-principles study," Phys. Rev. Lett., vol. 114, no. 11, pp. 115901, 2015.

[5] J. Bardeen, L. N. Cooper, and J. R. Schrieffer, "Theory of superconductivity," Phys. Rev., vol. 108, no. 5, pp. 1175-1204, 1957.

[6] R. van Leeuwen, "First-principles approach to the electron-phonon interaction," Phys. Rev. B, vol. 69, no. 11, pp. 115110, 2004.

[7] J. S. Jin and J. S. Lee, "Electron-phonon interaction model and prediction of thermal energy transport in SOI transistor," J. Nanosci. Nanotechnol., vol. 7, no. 11, pp. 4094-4100, 2007.

[8] J. S. Jin and J. S. Lee, "Electron-phonon interaction model and its application to thermal transport simulation during electrostatic discharge event in NMOS transistor," J. Heat Trans.-T. ASME, vol. 131, no. 9, pp. 092401, 2009.

[9] G. Qin, Z. Qin, S.-Y. Yue, Q.-B. Yand, and M. Hu, "External electric field driving the ultra-low thermal conductivity of silicone," Nanoscale, vol. 9, no. 21, pp. 7227-7234, 2017.

[10] J. S. Jin, B. J. Lee, and H. J. Lee, "Analysis of phonon transport in silicon nanowires including optical phonons," J. Korean Phys. Soc., vol. 63, no. 5, pp. 1007-1013, 2013.

[11] J. S. Jin, "Prediction of phonon and electron contributions to thermal conduction in doped silicon films," J. Mech. Sci. Technol., vol. 28, no. 6, pp. 2287-2292, 2014.

[12] S. V. J. Narumanchi, "Simulations of heat transport in sub-micron conduction," Ph.D. thesis, Mechanical Engineering, Carnegie Mellon University, Pittsburgh, PA, 2003.

[13] J. R. Barker, "Quantum transport theory of highfield conduction in semiconductors," J. Phys. C: Solid State Phys., vol. 6, no. 17, pp. 2663-2684, 1973.
[14] N. Sano and A. Yoshii, "Quantum effects on electron-phonon interaction under high electric field in semiconductors," Semicond. Sci. Technol., vol. 7, no. 3B, pp. B36-B38, 1992.

[15] J. Lai and A. Majumdar, "Concurrent thermal and electrical modeling of sub-micrometer silicon devices," J. Appl. Phys., vol. 79, no. 9, pp. 73537361, 1996.

[16] R. F. Pierret, Advanced Semiconductor Fundamentals, 2nd ed. Pearson Education, Inc., 2003.

[17] E. Pop, R. W. Dutton, and K. E. Goodson, "Analytic band Monte Carlo model for electron transport in Si including acoustic and optical phonon dispersion," J. Appl. Phys., vol. 96, no. 9, pp. 4998-5005, 2004.

[18] S. V. J. Narumanchi, J. Y. Murthy, and C. H. Amon, "Comparison of different phonon transport models for predicting heat conduction in silicon-oninsulator transistor," J. Heat Trans.-T. ASME, vol. 127, no. 7, pp. 713-723, 2005.

[19] S. Mazumder and A. Majumdar, "Monte Carlo study of phonon transport in solid thin films including dispersion and polarization," J. Heat Trans.-T. ASME, vol. 123, no. 4, pp. 749-759, 2001.

[20] J. C. Chai, H. S. Lee and S. V. Patankar, "Finite volume method for radiation heat transfer," $J$. Thermophys. Heat Transfer, vol. 8, no. 3, pp. 419-425, 1994.

[21] J. Y. Murthy and S. R. Mathur, "Finite volume method for radiative heat transfer using unstructured meshes," J. Thermophys Heat Tr., vol. 12, no. 3, pp. 313-321, 1998.

[22] S. V. J. Narumanchi, J. Y. Murthy, and C. H. Amon, "Submicron heat transport model in silicon accounting for phonon dispersion and polarization," J. Heat Trans.-T. ASME, vol. 126, no. 6, pp. 946955, 2004.

[23] D. K. Ferry, Semiconductor Transport. Taylor and Francis, 2000.

[24] H. Goldstein, Classical Mechanics, 2nd ed. AddisonWesley, 1980.

[25] E. Pop, J. Rowlette, R. W. Dutton, and K. E. Goodson, "Joule heating under quasi-ballistic transport conditions in bulk and strained silicon devices," in International Conference Simulation of Semiconductor Processes and Devices (SISPAD), Tokyo, 2005, pp. 307-310.

[26] J. S. Jin, "Electron-phonon interaction model and its application to numerical analysis of micro/nanoscale energy transport in silicon devices," Ph.D. thesis, ME, SNU, Seoul, 2007. 


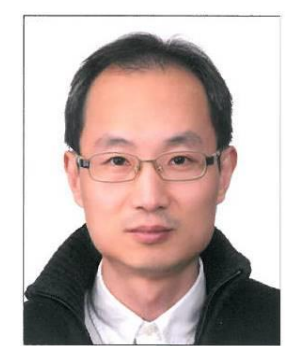

micro/nanoscales.
Jae Sik Jin received the B.S. degree in Department of Mechanical Design Engineering from Seoul National University of Science and Technology, the M.S degree in Mechanical Engineering from Hang Yang University, and the Ph.D. degree in School of Mechanical and Aerospace Engineering, Seoul National University, Korea. From 2009 to 2011, he was a Post Dr. with the Massachusetts Institute of Technology and Saint Louis University. After spending about 3 years at Samsung SDI as an engineer, he has been an Assistant Professor at the Department of Mechanical Design, Chosun College of Science and Technology since 2015. His research interests are in the understanding of fundamental energy transport and energy conversion mechanisms at 